\title{
Boundary integral based graded element for elastic analysis of 2D functionally graded plates
}

\author{
Hui Wang ${ }^{\mathrm{a}}$, Qing-Hua Qin ${ }^{\mathrm{b}, *}$ \\ ${ }^{a}$ Institute of Scientific and Engineering Computation, Henan University of Technology, Zhengzhou 450001, PR China \\ ${ }^{\mathrm{b}}$ Research School of Engineering, Australian National University, Canberra ACT0200, Australia
}

\section{A R T I C L E I N F O}

\section{Article history:}

Received 5 September 2011

Accepted 29 October 2011

Available online 15 November 2011

\section{Keywords:}

Functionally graded material

Fundamental solution

Graded element

Hybrid finite element

Boundary integral

\begin{abstract}
A B S T R A C T
A two-dimensional (2D) elastic analysis of functionally graded materials (FGMs) is conducted using the proposed boundary integral based graded element formulation. The graded element model is based on independent internal and frame field approximations. The elemental stiffness contains element boundary integrals only and is calculated using the exact expression of the graded material property. In the construction of the element model, the fundamental solutions of functionally graded plate with quadratic variation of elastic properties are employed to construct the internal approximation and then the graded element is constructed, in which the material definition entails naturally graded variation. Three numerical examples are considered: verification of fundamental solutions, a functionally graded cantilever beam, and a functionally graded link bar, to assess the performance of the hybrid graded model and to show the advantages of FGMs over homogeneous materials.
\end{abstract}

(c) 2011 Elsevier Ltd. All rights reserved.

\section{Introduction}

FGMs are a class of relatively new and promising composite materials that have optimized material properties by combining different material components following a predetermined law. They are heterogeneous composite materials with graded variation of constituents from one material phase to another, which results in continuously varying material properties. FGMs thus have superior thermal and mechanical performance to conventional homogeneous materials, and have a wide variety of engineering applications especially for the purpose of removing mismatches of thermo-mechanical properties between coating and substrate and reducing stress level in structures.

Because of the importance of FGMs, their thermo-mechanical behavior has been investigated by many researchers. In the context of numerical analysis, Reddy presented a finite element model based on the third-order shear deformation plate theory for the analysis of functionally graded plates (Reddy, 2000). Carrera et al. employed the concept of virtual displacements to obtain finite element solutions of functionally graded plates subjected to transverse mechanical loadings (Carrera et al., 2008). Kim and Paulino developed a graded finite element model within the framework of a generalized isoparametric formulation (Kim and Paulino, 2002).

\footnotetext{
* Corresponding author. Tel.: +612 61258274; fax: +61261250506

E-mail address: qinghua.qin@anu.edu.au (Q.-H. Qin).
}

Ching and Yen extended the meshless local Petrov-Galerkin (MLPG) method for thermo-elastic analysis of FGMs (Ching and Yen, 2005). Wang and Qin investigated the thermo-elastic and heat conduction performance of inhomogeneous graded materials using the mixed meshless method coupled with fundamental solutions of homogeneous materials and globally supported radial basis functions (Wang and Qin, 2008; Wang et al., 2005, 2006). Furthermore, the enriched finite element method (FEM) adding crack-tip fields to the element interpolation (Ayhan, 2009), the method of fundamental solutions (MFS) (Marin and Lesnic, 2007), the meshless local boundary integral equation (LBIE) method (Sladek et al., 2005), the boundary element method (BEM) (Sutradhar and Paulino, 2004; Zhang et al., 2011), the modified smoothed particle hydrodynamics (MSPH) method (Zhang and Batra, 2007), the element-free Galerkin method (EFGM) (Dai et al., 2005), and so on, have also been proposed to conduct temperature, displacement and stress estimation in FGM-based structures.

As an alternative to the numerical methods mentioned above, a hybrid finite element method with fundamental solutions as intraelement interpolation functions was presented for analyzing heat conduction problems (Wang and Qin, 2009, 2010a) and elastic problems (Wang and Qin, 2010b, 2011). This model inherits the advantages of flexible domain element division in Trefftz-FEM (Qin, 2000) and boundary integrals in BEM (Qin, 1993), and due to the use of fundamental solutions, is referred to as HFS-FEM. In the HFS-FEM, the intra-element field is approximated by a linear combination of fundamental solutions in terms of sources (singularities) which are 
placed on an outer pseudo-boundary similar to the real element boundary. The field variables on the element boundary are independently defined by way of conventional shape functions. The linear equation system of unknowns can be established by the stationary condition of a modified variational functional.

In this study, a HFS-FEM model is developed for solving 2D elastic problems of FGMs with quadratic variation of material properties. The model is then used to study the advantages of FGM over homogeneous materials in terms of reducing stress reconstruction. In the development of the model, a weak formulation of HFS-FEM and its discrete system of equations are derived for the solution of functionally graded elastic problems and used to construct elaborately graded elements. In particular, the fundamental solutions corresponding to FGM with quadratic variation of material properties are employed to approximate the intra-element fields, so that the governing equations of the problem of interest are analytically satisfied within the element. Unlike the graded finite element (Kim and Paulino, 2002) and the conventional finite element (Ayhan, 2009) mentioned above, the material definition in the proposed graded element can retain its natural variation, without any approximation.

The paper is organized as follows. Section 2 gives the corresponding governing equation for functionally graded elastic plate and its fundamental solution. The FGM considered has a quadratic variation of material properties. In Section 3, the hybrid finite element formulations in which the fundamental solutions is taken as intra-element interpolation functions are derived to produce the final force-displacement stiffness equations. Numerical results are presented and analyzed in Section 4. Finally, Section 5 presents some conclusions.

\section{Formulation of the problem}

In this section, basic equations and the corresponding fundamental solutions for FGMs presented in (Yuan and Yin, 2011) are briefly reviewed to provide notations and references for the subsequent sections.

\subsection{Basic equations}

For a 2D linear elastic problem, the governing equations of force equilibrium in the absence of body forces are given by

$\sigma_{i j, j}=0$

where $\sigma_{i j}$ are the components of the Cauchy stress tensor. For plane problems, all indices range from 1 to 2 and an index followed by a comma stands for partial differentiation with respect to the spatial coordinate. The summation convention is implied for repeated indices.

For the functionally graded materials considered in this study, the elastic stiffness tensor $C_{i j k l}$ is associated with the spatial variable $\mathbf{x}=\left(x_{1}, x_{2}\right)$; that is, $C_{i j k l}=C_{i j k l}(\mathbf{x})$. Therefore, the linear elastic strainstress relation is written as

$\sigma_{i j}=C_{i j k l}(\mathbf{x}) \varepsilon_{k l}$

The components of stiffness tensor $C_{i j k l}$ must satisfy the usual symmetric condition

$C_{i j k l}=C_{k l i j}=C_{i j l k}=C_{j i k l}$

Specially, for isotropic inhomogeneous elastic media, the elastic stiffness tensor $C_{i j k l}$ is written as

$C_{i j k l}(\mathbf{x})=\lambda(\mathbf{x}) \delta_{i j} \delta_{k l}+\mu(\mathbf{x})\left(\delta_{i k} \delta_{j l}+\delta_{i l} \delta_{j k}\right)$ where $\delta_{i j}$ is Kronecker's delta, the Lame elastic parameters $\lambda(\mathbf{x})$ and $\mu(\mathbf{x})$ are the functions of spatial coordinate variable $\mathbf{x}$ and can be expressed in terms of elastic modulus $E$, and Poisson ratio $\nu$ as

$\lambda(\mathbf{x})=\frac{3-\kappa}{\kappa-1} \mu(\mathbf{x}), \quad \mu(\mathbf{x})=\frac{E(\mathbf{x})}{2(1+\nu)}$

with $\kappa=3-4 \nu$ for plane strain and $\kappa=(3-\nu) /(1+\nu)$ for plane stress.

Therefore, the constitutive law Eq. (2) can be simplified as

$\sigma_{i j}=\lambda(\mathbf{x}) \delta_{i j} \varepsilon_{k k}+2 \mu(\mathbf{x}) \varepsilon_{i j}$

As well, the infinitesimal strain tensor $\varepsilon_{i j}$ related to the displacement field is expressed as

$\varepsilon_{i j}=\frac{1}{2}\left(u_{i, j}+u_{j, i}\right)$

Substituting Eq. (7) into the constitutive Eq. (6) and then into the equilibrium Eq. (1) we have

$$
\begin{aligned}
& \lambda_{, i}(\mathbf{x}) u_{j, j}(\mathbf{x})+\{\lambda(\mathbf{x})+\mu(\mathbf{x})\} u_{j, j i}(\mathbf{x})+\mu(\mathbf{x}) u_{i, j j}(\mathbf{x})+\mu_{, j}(\mathbf{x})\left\{u_{i, j}(\mathbf{x})\right. \\
& \left.\quad+u_{j, i}(\mathbf{x})\right\}=0
\end{aligned}
$$

If the material is homogeneous, i.e., the Lame parameters are independent of the spatial variable $\mathbf{x}$, Eq. (8) becomes

$\{\lambda+\mu\} u_{j, j i}(\mathbf{x})+\mu u_{i, j j}(\mathbf{x})=0$

which is the classic Navier-Cauchy equation with respect to displacements.

The boundary conditions have the same form as those of homogeneous materials:

$u_{i}=\bar{u}_{i}$ on $\Gamma_{u}$

$t_{i}=\overline{t_{i}}$ on $\Gamma_{t}$

where $t_{i}=\sigma_{i j} n_{j}$ represents the $i$ th component of the boundary traction, and $n_{i}$ is the $i$ th component of outward normal to the boundary. $\Gamma_{u}$ and $\Gamma_{t}$ are the boundaries on which the displacement and the traction are prescribed respectively. An overbar denotes that the variable is specified.

\subsection{Fundamental solutions for quadratic variation of elasticity}

In this work, the Lame constants $\lambda$ and $\mu$ are assumed to be quadratic variation of the spatial variable $\mathbf{x}$, that is

$\lambda(\mathbf{x})=\lambda_{0}\left(c+\beta_{i} x_{i}\right)^{2}, \quad \mu(\mathbf{x})=\mu_{0}\left(c+\beta_{i} x_{i}\right)^{2}$

where $c, \lambda_{0}$ and $\mu_{0}$ are the corresponding material constants, $\beta_{i}$ is a graded parameter, which has a dimension of $\mathrm{m}^{-1}$. In particular, if the graded parameter $\beta_{i}$ is equal to zero, the Lame constants in Eq. (11) will be reduced to two constants, and then the system of partial differential Eq (8) will be the standard Navier-Cauchy equations for homogeneous isotropic elastic materials.

According to the work of (Manolis and Shaw, 1996), when the Poisson ratio $\nu$ is equal to 0.25 (a rather common value for rock materials) and the plane strain state is considered, one obtains

$\lambda_{0}=\mu_{0}$

which can significantly simplify the derivation of fundamental solutions.

Generally, the free space fundamental displacement solution for an isotropic inhomogeneous elastic continuum must satisfy the following equation system 


$$
\begin{aligned}
& \lambda_{, i}(\mathbf{x}) u_{j, j}(\mathbf{x})+\{\lambda(\mathbf{x})+\mu(\mathbf{x})\} u_{j, j i}(\mathbf{x})+\mu(\mathbf{x}) u_{i, j j}(\mathbf{x})+\mu_{, j}(\mathbf{x})\left\{u_{i, j}(\mathbf{x})\right. \\
& \left.\quad+u_{j, i}(\mathbf{x})\right\}+\delta\left(\mathbf{x}-\mathbf{x}^{S}\right) e_{i}=0
\end{aligned}
$$

where $\mathbf{x}$ is a field point in the infinite plane, $\mathbf{x}^{S}$ is a source point at which the unit force $e_{i}$ along the $i$-direction is applied, and $\delta(\mathbf{x})$ is the Dirac delta function.

To obtain the fundamental displacement solution for the equilibrium Eq (13) the following transformation is established for the displacement vector (Yuan and Yin, 2011)

$w_{i}=\left(c+\beta_{k} x_{k}\right) u_{i}$

from which we have

$u_{i, j}=\frac{w_{i, j}}{c+\beta_{k} x_{k}}-\frac{w_{i} \beta_{j}}{\left(c+\beta_{k} x_{k}\right)^{2}}$

and then the stress component can be given by

$\sigma_{i j}=\left(c+\beta_{l} x_{l}\right)\left[\lambda_{0} \delta_{i j} w_{k, k}+\mu_{0}\left(w_{i, j}+w_{j, i}\right)\right]-\left[\lambda_{0} \delta_{i j} w_{k} \beta_{k}\right.$

$$
\left.+\mu_{0}\left(w_{i} \beta_{j}+w_{j} \beta_{i}\right)\right]
$$

Substituting Eqs. (14) and (11) into Eq. (13), we obtain

$2 \mu_{0} w_{j, j i}+\mu^{0} w_{i, j j}+\frac{1}{\left(c+\beta_{k} x_{k}\right)} \delta\left(\mathbf{x}-\mathbf{x}^{s}\right) e_{i}=0$

If the concentrated force acts at the origin, using the logarithmic potential, Yuan and Yin (2011) obtain

$w_{l i}^{*}=\frac{1}{6 \pi \mu_{0}}\left\{-2 \delta_{l i} \ln r+\frac{x_{i} x_{l}}{r^{2}}\right\}$

where $w_{l i}^{*}$ denote the generalized displacement solutions at the field point $\mathbf{x}$ along the $i$-direction when a unit point force is applied at the origin along the $l$-direction. After this, with the inverse transformation of Eq. (14), and at the same time moving the point force from the origin to an arbitrary source point $\mathbf{x}_{S}$, the displacement components can be written as

$u_{l i}^{*}=\frac{c}{6 \pi \mu_{0}\left(c+\beta_{k} x_{k}\right)\left(c+\beta_{k} x_{k}^{s}\right)}\left\{-2 \delta_{l i} \ln r+\frac{r_{i} r_{l}}{r^{2}}\right\}$

where some useful quantities related to the distance $r$ are

$r=\left(r_{i} r_{i}\right)^{1 / 2}$

$r_{i}=x_{i}-x_{i}^{S}$

$r_{, i}=\frac{r_{i}}{r}$

$r_{i} r_{i}=1$

$r_{i, j}=\delta_{i j}$

Based on the displacement formulation (19) (Yuan and Yin, 2011), we obtain the strain components by differentiating the solution (19) with respect to the spatial variable $x_{j}$

$$
\begin{aligned}
\varepsilon_{l i j}^{*}= & -\frac{c}{12 \pi \mu_{0}\left(c+\beta_{k} x_{k}\right)^{2}\left(c+\beta_{k} x_{k}^{s}\right)}\left\{\beta_{j}\left(-2 \delta_{i l} \ln r+\frac{r_{i} r_{l}}{r^{2}}\right)\right. \\
& \left.+\beta_{i}\left(-2 \delta_{j l} \ln r+\frac{r_{j} r_{l}}{r^{2}}\right)\right\} \\
& +\frac{c}{12 \pi \mu_{0}\left(c+\beta_{k} x_{k}\right)\left(c+\beta_{k} x_{k}^{s}\right)}\left\{\frac{2 \delta_{i j} r_{l}-\delta_{i l} r_{j}-\delta_{j l} r_{i}}{r^{2}}-\frac{4 r_{i} r_{l} r_{j}}{r^{4}}\right\}
\end{aligned}
$$

and then, the stress components are given by

$$
\begin{aligned}
\sigma_{l i j}^{*}= & \frac{c}{6 \pi\left(c+\beta_{k} x_{k}^{s}\right)}\left\{\left[\left(2 \beta_{l} \ln r-\frac{\beta_{k} r_{k} r_{l}}{r^{2}}\right)-\left(c+\beta_{k} x_{k}\right) \frac{r_{l}}{r^{2}}\right] \delta_{i j}\right. \\
& +\beta_{j}\left(2 \delta_{i l} \ln r-\frac{r_{i} r_{l}}{r^{2}}\right)+\beta_{i}\left(2 \delta_{j l} \ln r-\frac{r_{j} r_{l}}{r^{2}}\right)+\left(c+\beta_{k} x_{k}\right) \\
& \left.\times\left(\frac{2 \delta_{i j} r_{l}-\delta_{i l} r_{j}-\delta_{j l} r_{i}}{r^{2}}-\frac{4 r_{i} r_{l} r_{j}}{r^{4}}\right)\right\}
\end{aligned}
$$

It is obvious that the fundamental solutions (19) and (22) can easily be reduced to the homogeneous fundamental solutions, when the graded parameters $\beta_{i}=0(i=1,2)$ and $c=1$. For example, for homogeneous isotropic materials with Poisson ratio $\nu=0.25$, $\lambda=\lambda_{0}=\mu=\mu_{0}$, we have

$u_{l i}^{*}=\frac{1}{6 \pi \mu_{0}}\left[-2 \delta_{i l} \ln r+\frac{r_{l} r_{i}}{r^{2}}\right]$

and

$\sigma_{\text {lij }}^{*}=\frac{1}{6 \pi}\left[\frac{r_{l} \delta_{i j}-r_{j} \delta_{i l}-r_{i} \delta_{j l}}{r^{2}}-\frac{4 r_{i} r_{j} r_{l}}{r^{4}}\right]$

which are same as the formulations used in BEM for homogeneous materials.

\section{Hybrid finite element formulation}

\subsection{Hybrid functional and element stiffness equation}

The initial concept of the hybrid finite element method features two independent fields (interior and frame fields) assumed in a given element. In the present work, the variables $u_{i}$ and $\tilde{u}_{i}$ respectively represent the interior and frame field variables. In the absence of body forces, the variational functional for any given element, say element $e$, used in the present model can be constructed as (Wang and Qin, 2010b, 2011)

$\Pi_{m e}=\frac{1}{2} \int_{\Omega_{e}} \sigma_{i j} \varepsilon_{i j} \mathrm{~d} \Omega-\int_{\Gamma_{\text {te }}} \overline{t_{i}} \tilde{u}_{i} \mathrm{~d} \Gamma+\int_{\Gamma_{e}} t_{i}\left(\tilde{u}_{i}-u_{i}\right) \mathrm{d} \Gamma$

where $\Omega_{e}$ is the domain of element e, $\Gamma_{t e}$ and $\Gamma_{u e}$ are boundaries, where the traction and displacement are respectively specified, and $\Gamma_{e}$ denotes the whole element boundary. The inter-element boundary is denoted by $\Gamma_{I e}$. Clearly, for the hybrid element shown in Fig. 1 we have

$\Gamma_{e}=\Gamma_{u e} \cup \Gamma_{t e} \cup \Gamma_{\text {Ie }}$

Making use of Gauss theorem, the first-order variational of the functional can be further written as

$$
\begin{aligned}
\delta \Pi_{m e}=- & \int_{\Omega_{e}} \sigma_{i j, j} \delta u_{i} \mathrm{~d} \Omega+\int_{\Gamma_{l e}} t_{i} \delta \tilde{u}_{i} \mathrm{~d} \Gamma+\int_{\Gamma_{t e}}\left(t_{i}-\bar{t}_{i}\right) \delta \tilde{u}_{i} \mathrm{~d} \Gamma \\
& +\int_{\Gamma_{e}} \delta t_{i}\left(\tilde{u}_{i}-u_{i}\right) \mathrm{d} \Gamma
\end{aligned}
$$

in which the first integral gives the equilibrium equation $\sigma_{i j, j}=0$, and the second integral enforces the reciprocity condition by coconsidering those from neighboring elements. The traction boundary condition can be enforced by the third integral, and the final integral enforces equality of $u_{i}$ and $\tilde{u}_{i}$ along the elemental frame boundary $\Gamma_{e}$. 


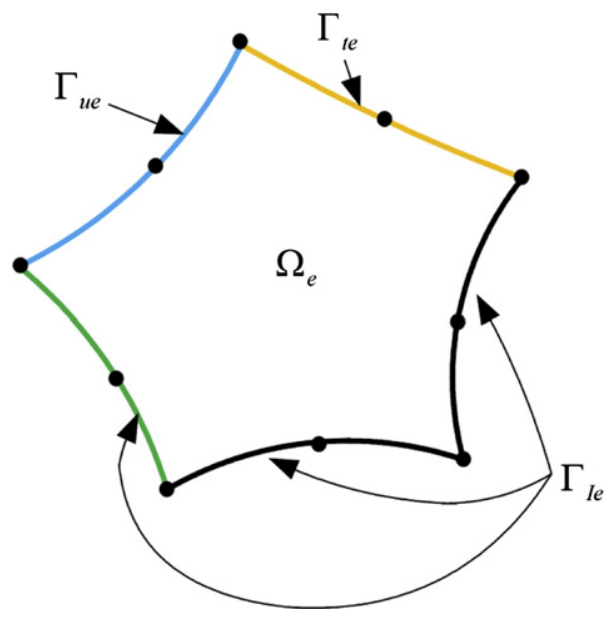

Fig. 1. Schematic of the hybrid element.

In the present hybrid formulation, in order to obtain the element stiffness equation involving element boundary integrals only, the element interior displacement field is approximated by the linear combination of the fundamental solutions at a series of source points $\mathbf{x}_{m}^{s}$ located outside the element domain as

$u_{i}(\mathbf{x})=c_{l m} u_{l i}^{*}\left(\mathbf{x}, \mathbf{x}_{m}^{s}\right)=\left[\mathbf{N}_{i}\right]\left\{\mathbf{c}_{e}\right\} \quad i, l=1,2, m=1,2, \ldots, M$

where $M$ is the number of virtual sources outside the element domain, $\left\{\mathbf{c}_{e}\right\}=\left[\begin{array}{lllll}c_{11} & c_{21} & \cdots & c_{1 M} & c_{2 M}\end{array}\right]^{\mathrm{T}}$ is an unknown coefficient vector (not nodal displacement), and the interpolation matrix

$$
\left[\mathbf{N}_{i}\right]=\left[\begin{array}{lllll}
u_{1 i}^{*}\left(\mathbf{x}, \mathbf{x}_{1}^{S}\right) & u_{2 i}^{*}\left(\mathbf{x}, \mathbf{x}_{1}^{S}\right) & \cdots & u_{1 i}^{*}\left(\mathbf{x}, \mathbf{x}_{M}^{S}\right) & u_{2 i}^{*}\left(\mathbf{x}, \mathbf{x}_{M}^{S}\right)
\end{array}\right]
$$

consists of the fundamental solutions $u_{l i}^{*}\left(\mathbf{x}, \mathbf{x}^{s}\right)$ at $M$ source points.

It is noted that the constructed displacement field (28) can analytically satisfy the inhomogeneous elastic governing Eq. (13), since the fundamental solutions (19) of the problem are used as the interpolation functions.

Making use of the strain-displacement Eq. (7) and the stressstrain relationship (2), the corresponding stress and traction components are expressed as

$$
\begin{aligned}
\sigma_{i j}(\mathbf{x}) & =c_{l m} \sigma_{l i j}^{*}\left(\mathbf{x}, \mathbf{x}_{m}^{s}\right)=\left[\mathbf{S}_{i j}\right]\left\{\mathbf{c}_{e}\right\} \quad i, j, l=1,2, \\
m & =1,2, \ldots, M
\end{aligned}
$$

and

$t_{i}(\mathbf{x})=c_{l m} t_{l i}^{*}\left(\mathbf{x}, \mathbf{x}_{m}^{S}\right)=\left[\mathbf{Q}_{i}\right]\left\{\mathbf{c}_{e}\right\} \quad i, l=1,2, \quad m=1,2, \ldots, M$

in which

$$
\begin{aligned}
& {\left[\mathbf{S}_{i j}\right]=\left[\begin{array}{lllll}
\sigma_{1 i j}^{*}\left(\mathbf{x}, \mathbf{x}_{1}^{S}\right) & \sigma_{2 i j}^{*}\left(\mathbf{x}, \mathbf{x}_{1}^{S}\right) & \cdots & \sigma_{1 i j}^{*}\left(\mathbf{x}, \mathbf{x}_{M}^{S}\right) & \sigma_{2 i j}^{*}\left(\mathbf{x}, \mathbf{x}_{M}^{S}\right)
\end{array}\right]}
\end{aligned}
$$

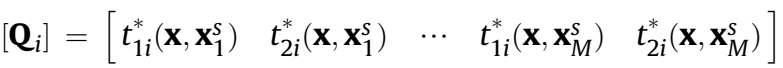

with the traction kernels being defined by

$t_{l i}^{*}\left(\mathbf{x}, \mathbf{x}_{1}^{S}\right)=\sigma_{l i j}^{*}\left(\mathbf{x}, \mathbf{x}_{m}^{S}\right) n_{j}$
To enforce conformity of the displacement field on the common interface of any two neighboring elements, frame displacement fields $\tilde{u}_{i}$ are separately assumed on the element boundary as

$\tilde{u}_{i}(\mathbf{x})=d_{l k} \tilde{N}_{l i}\left(\mathbf{x}, \mathbf{x}_{k}\right)=\left[\tilde{\mathbf{N}}_{i}\right]\left\{\mathbf{d}_{e}\right\} \quad i, l=1,2, \quad k=1,2, \ldots, K$

where $\left[\tilde{\mathbf{N}}_{i}\right]$ denotes the interpolation vector relating the boundary displacement to the nodal displacement vector $\left\{\mathbf{d}_{e}\right\}$.

To obtain the element stiffness equation and the optional relationship of unknown coefficient $\left\{\mathbf{c}_{e}\right\}$ and $\left\{\mathbf{d}_{e}\right\}$, the application of Gauss theorem to the functional (25) gives

$\Pi_{m e}=-\frac{1}{2} \int_{\Omega_{e}} \sigma_{i j, j} u_{i} \mathrm{~d} \Omega-\frac{1}{2} \int_{\Gamma_{e}} t_{i} u_{i} \mathrm{~d} \Gamma-\int_{\Gamma_{\text {te }}} \bar{t}_{i} \tilde{u}_{i} \mathrm{~d} \Gamma+\int_{\Gamma_{e}} s_{i} \tilde{u}_{i} \mathrm{~d} \Gamma$

Because the assumed displacement field (28) and stress field (30) analytically satisfy the governing Eq. (13), we have

$\Pi_{m e}=-\frac{1}{2} \int_{\Gamma_{e}} t_{i} u_{i} \mathrm{~d} \Gamma-\int_{\Gamma_{t e}} \bar{t}_{i} \tilde{u}_{i} \mathrm{~d} \Gamma+\int_{\Gamma_{e}} t_{i} \tilde{u}_{i} \mathrm{~d} \Gamma$

Substituting Eqs. (28), (31) and (35) into the functional (37) yields

$\Pi_{m e}=-\frac{1}{2}\left\{\mathbf{c}_{e}\right\}^{\mathrm{T}}\left[\mathbf{H}_{e}\right]\left\{\mathbf{c}_{e}\right\}-\left\{\mathbf{d}_{e}\right\}^{\mathrm{T}}\left\{\mathbf{g}_{e}\right\}+\left\{\mathbf{c}_{e}\right\}^{\mathrm{T}}\left[\mathbf{G}_{e}\right]\left\{\mathbf{d}_{e}\right\}$

where

$$
\begin{aligned}
{\left[\mathbf{H}_{e}\right] } & =\int_{\Gamma_{e}}\left[\mathbf{Q}_{i}\right]^{\mathrm{T}}\left[\mathbf{N}_{i}\right] \mathrm{d} \Gamma \\
{\left[\mathbf{G}_{e}\right] } & =\int_{\Gamma_{e}}\left[\mathbf{Q}_{i}\right]^{\mathrm{T}}\left[\tilde{\mathbf{N}}_{i}\right] \mathrm{d} \Gamma \\
\left\{\mathbf{g}_{e}\right\} & =\int_{\Gamma_{\text {te }}} \bar{t}_{i}\left[\tilde{\mathbf{N}}_{i}\right]^{\mathrm{T}} \mathrm{d} \Gamma
\end{aligned}
$$

The stationary condition of the functional (38) with respect to $\left\{\mathbf{c}_{e}\right\}$ and $\left\{\mathbf{d}_{e}\right\}$ yields, respectively, the optional relationship between $\left\{\mathbf{c}_{e}\right\}$ and $\left\{\mathbf{d}_{e}\right\}$ and the element stiffness equation as

$\left\{\mathbf{c}_{e}\right\}=\left[\mathbf{H}_{e}\right]^{-1}\left[\mathbf{G}_{e}\right]\left\{\mathbf{d}_{e}\right\}$

and

$\left[\mathbf{K}_{e}\right]\left\{\mathbf{d}_{e}\right\}=\left\{\mathbf{g}_{e}\right\}$

with

$\left[\mathbf{K}_{e}\right]=\left[\mathbf{G}_{e}\right]^{\mathbf{T}}\left[\mathbf{H}_{e}\right]^{-1}\left[\mathbf{G}_{e}\right]$

being the element stiffness matrix, which is sparse and symmetric.

\subsection{Naturally graded element}

From the hybrid formulation described above we can see that the independence of intra-element displacement fields and boundary displacements makes it possible to construct arbitrarily shaped hybrid elements, as the continuity conditions between elements are required on the element boundary (frame fields) only. Moreover, because the assumed intra-element displacements analytically satisfy the inhomogeneous elastic governing Eq. (13) within the element domain, it is possible to design special largescale elements with arbitrary element edges and multiple nodes 
along each element edge to reduce the meshing effort for problems with irregular geometry (Wang and Qin, 2011).

In the present work, a naturally graded element in which the material definition naturally accords with the specified graded variation is developed, because the related fundamental solutions are used for the intra-element displacement and stress approximations. Fig. 2 displays a general eight-node hybrid element with four edges and three nodes at each edge. For the specified hybrid element, the independent interior and frame fields are incorporated by the functional (38) at element level. In the computational process, a local coordinate system $\left(\tilde{x}_{1}, \tilde{x}_{2}\right)$ with the origin centered in the element and axis parallel to the global axis is introduced, and thus the material definition for the specified graded element should be modified, i.e. if the global coordinate of the centroid of the element is $\mathbf{x}_{c}\left(x_{1 c}, x_{2 c}\right)$, then the relation of the local and global coordinate systems is written as

$x_{i}=x_{i c}+\tilde{x}_{i}$

The material definitions such as $\mu$ can then be rewritten within the element as

$\mu=\mu_{0}\left(c+\beta_{i} x_{i}\right)^{2}=\mu_{0}\left(c+\beta_{i} x_{i c}+\beta_{i} \tilde{x}_{i}\right)^{2}=\mu_{0}\left(\tilde{c}+\beta_{i} \tilde{x}_{i}\right)^{2}$

with $\tilde{c}=c+\beta_{i} x_{i c}$.

Further, in the proposed hybrid element, the singularities of the fundamental solution can be avoided by placing the related source points on a pseudo-boundary which has a similar shape to the element physical boundary but is outside the element domain, as was done in the MFS (Fairweather and Karageorghis, 1998; Wang and Qin, 2008; Wang et al., 2006; Young et al., 2006). In the present work, these source points are generated by means of the relation

$\mathbf{x}^{S}=\mathbf{x}_{c}+\gamma \mathbf{x}_{b}$

where $\mathbf{x}^{s}$ and $\mathbf{x}_{b}$ are, respectively, the source point and boundary node, and $\mathbf{x}_{c}$ denotes the element centroid, as shown in Fig. 2 . The dimensionless parameter $\gamma$ has the function of adjusting the distance between the pseudo-boundary and the physical element boundary. Small value of $\gamma$ means that the pseudo-boundary closes to the element boundary very much. For this case, the near singularity of fundamental solutions may have a negative effect to the numerical accuracy. Conversely, if the parameter $\gamma$ is too large, or the pseudo-boundary is far from the element boundary, source points usually have larger values and thus the numerical round-off error caused by large magnitude difference between unknown $\boldsymbol{c}_{\mathrm{e}}$

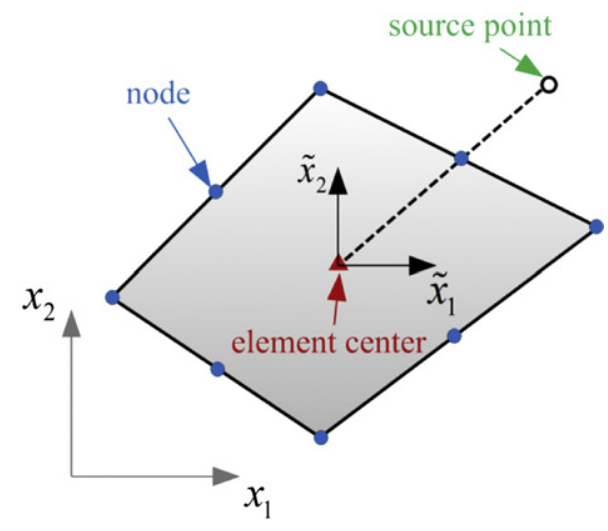

Fig. 2. Eight-node hybrid graded element. and $\boldsymbol{d}_{\mathrm{e}}$ also leads to the decrease of numerical accuracy. According to the authors' previous works, the parameter $\gamma$ can be chosen in the range $3<\gamma<10$ to achieve stable and reliable results (Wang and Qin, 2009, 2010a,b, 2011). Here, the value of $\gamma$ is chosen to be 5.

\section{Results and discussion}

Based on the formulation described in Section 3, computer code was written in Matlab to perform deformation and stress analysis of 2D functionally graded plates under mechanical loads. For the sake of simplification, only unidirectional variation of an elastic parameter is considered in this work, i.e. if we set the graded parameter $\beta_{1}$ to be zero and simultaneously let $c=1$, then the remaining parameter $\beta_{2}$ can be evaluated by considering the elastic moduli of the constituent phases of the functionally graded medium. For example, if phase 1 and phase 2 have respectively elastic modules $E_{1}$ and $E_{2}$ (see Fig. 3), we have

$$
\begin{aligned}
& E(a)=2.5 \mu_{0}\left(c+\beta_{2} a\right)^{2}=E_{1} \\
& E(b)=2.5 \mu_{0}\left(c+\beta_{2} b\right)^{2}=E_{2}
\end{aligned}
$$

which leads to

$\beta_{2}=\frac{c\left(\sqrt{E_{1}}-\sqrt{E_{2}}\right)}{a \sqrt{E_{2}}-b \sqrt{E_{1}}}, \quad \mu_{0}=\frac{E_{1}}{2.5\left(c+\beta_{2} a\right)^{2}}$

To assess the performance and applicability of the proposed element model, three examples are considered in this section, namely a functionally graded circular plate, a cantilever beam, and a link bar. The results obtained from the proposed element formulation are compared with the available analytical solutions or numerical solutions from the commercial software ABAQUS using a refined finite mesh. In ABAQUS there is no specified material module for the direct analysis of functionally graded plate. To achieve results with acceptable accuracy the functionally graded medium to be analyzed is usually divided into a number of layers, each layer having a different material definition, with constant elastic parameters which are taken from the value at the element centroid (see Fig. 4). As a result, a step-like material property is assumed (see the vertical dashed lines in Fig. 4). Theoretically, when the number of material layers increases to infinity, the real quadratic curve of material property (see the solid curved line in Fig. 4) can be accurately represented.

\subsection{Verification of the proposed hybrid formulation}

To verify the proposed approach, an example of a circular plate is considered. The circular plate is in the state of plane strain and its radius is $R=10 \mathrm{~m}$ (see Fig. 5). The outer boundary of the circle is assumed to be fixed by constraining the two displacement

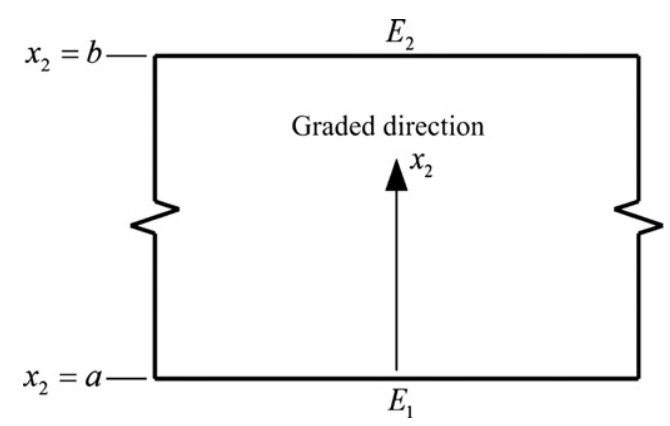

Fig. 3. Sketch of unidirectional variation of elastic parameter. 


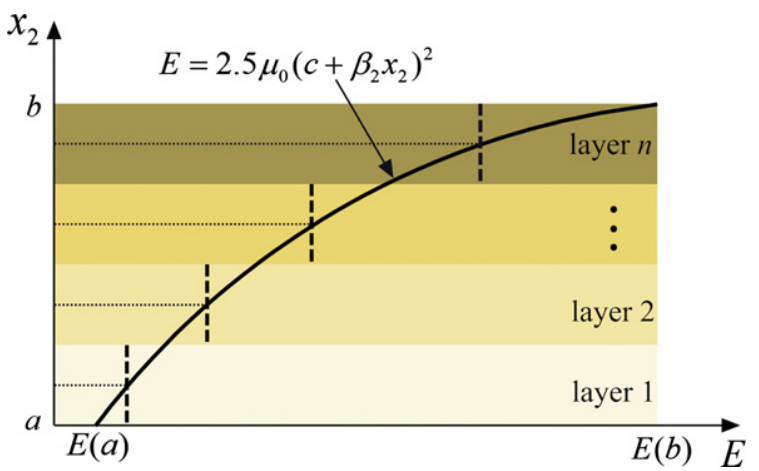

Fig. 4. Schematic of material definition in ABAQUS.

components. If a concentrated force $P_{2}=-10,000 \mathrm{~N}$ is applied at the center of the circle, the theoretical solutions of displacement are given by (Yuan and Yin, 2011) as

$$
\begin{aligned}
& u_{1}=\frac{1}{c+\beta_{k} x_{k}} \frac{P_{2}}{6 \pi \mu_{0}}\left(\frac{x_{1} x_{2}}{r^{2}}-\frac{x_{1} x_{2}}{R^{2}}\right) \\
& u_{2}=\frac{1}{c+\beta_{k} x_{k}} \frac{P_{2}}{6 \pi \mu_{0}}\left(-2 \ln \frac{r}{R}+\frac{x_{2}^{2}}{r^{2}}+\frac{x_{1}^{2}-R^{2}}{R^{2}}\right)
\end{aligned}
$$

from which we obtain the corresponding stress solutions as

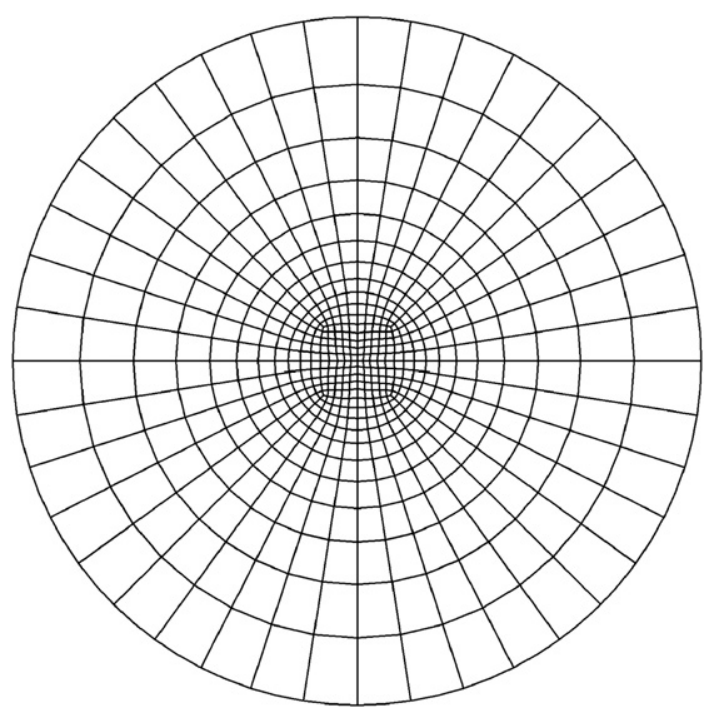

Fig. 6. Element mesh used in the present hybrid finite element model.

model the circle domain. Fig. 7 shows the vertical displacement along the axis $x_{2}=0$ (perpendicular to the gradation direction). Fig. 8 displays the vertical displacement along the axis $x_{1}=0$ (in the

$$
\begin{aligned}
& \sigma_{11}=\frac{P_{2}}{6 \pi}\left\{\left[-\frac{3 x_{2}}{R^{2}}+\frac{3 x_{2}}{r^{2}}-\frac{2 x_{2}\left(x_{2}^{2}+3 x_{1}^{2}\right)}{r^{4}}\right]\left(c+\beta_{k} x_{k}\right)+3 x_{1} x_{2}\left(\frac{1}{R^{2}}-\frac{1}{r^{2}}\right) \beta_{1}+\left(2 \ln \frac{r}{R}-\frac{x_{2}^{2}}{r^{2}}-\frac{x_{1}^{2}}{R^{2}}+1\right) \beta_{2}\right\} \\
& \sigma_{22}=\frac{P_{2}}{6 \pi}\left\{\left[-\frac{x_{2}}{R^{2}}+\frac{x_{2}}{r^{2}}-\frac{2 x_{2}\left(x_{1}^{2}+3 x_{2}^{2}\right)}{r^{4}}\right]\left(c+\beta_{k} x_{k}\right)+x_{1} x_{2}\left(\frac{1}{R^{2}}-\frac{1}{r^{2}}\right) \beta_{1}+\left(6 \ln \frac{r}{R}-\frac{3 x_{2}^{2}}{r^{2}}-\frac{3 x_{1}^{2}}{R^{2}}+3\right) \beta_{2}\right\} \\
& \sigma_{12}=\frac{P_{2}}{6 \pi}\left\{\left[\frac{x_{1}}{R^{2}}-\frac{x_{1}}{r^{2}}-\frac{4 x_{1} x_{2}^{2}}{r^{4}}\right]\left(c+\beta_{k} x_{k}\right)+\left(2 \ln \frac{r}{R}-\frac{x_{2}^{2}}{r^{2}}-\frac{x_{1}^{2}}{R^{2}}+1\right) \beta_{1}+x_{1} x_{2}\left(\frac{1}{R^{2}}-\frac{1}{r^{2}}\right) \beta_{2}\right\}
\end{aligned}
$$

In the analysis, the material parameters are assumed as follows: $c=1, \beta_{1}=0, \beta_{2}=0.1 \mathrm{~m}^{-1}$ and $\mu_{0}=2 \times 10^{7} \mathrm{~Pa}$. In total 900 hybrid elements with 2471 nodes, as shown in Fig. 6, are used to

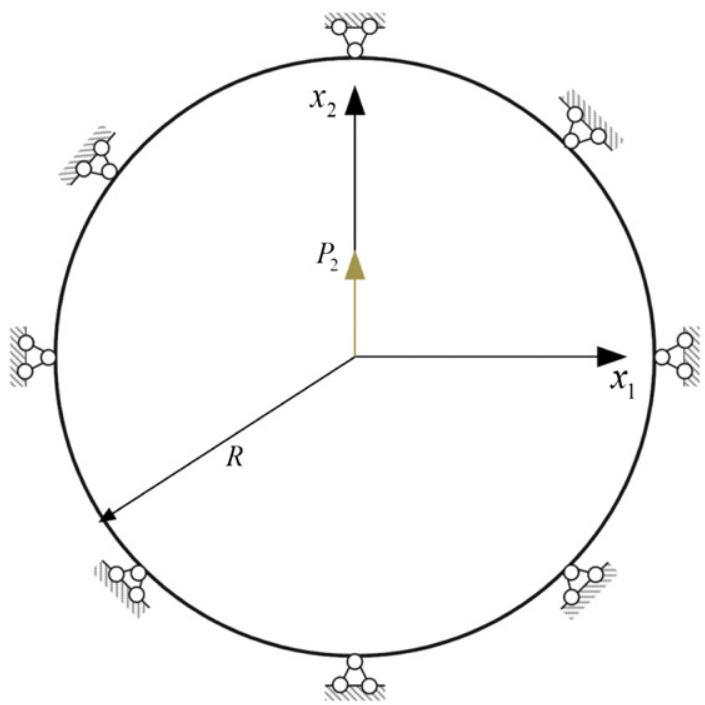

Fig. 5. A circle subjected to a centered concentrated force. gradation direction). Good agreement between the theoretical solution and the numerical results from the HFS-FEM is observed. It can also be found that when the material property varies with $x_{2}$

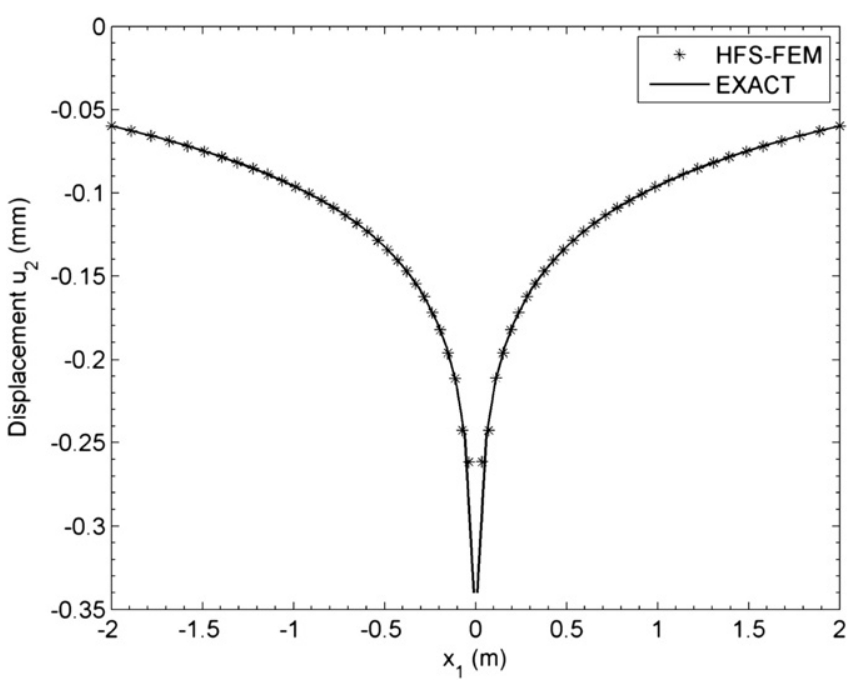

Fig. 7. Variation of displacement $u_{2}$ along the axis $x_{2}=0$ 


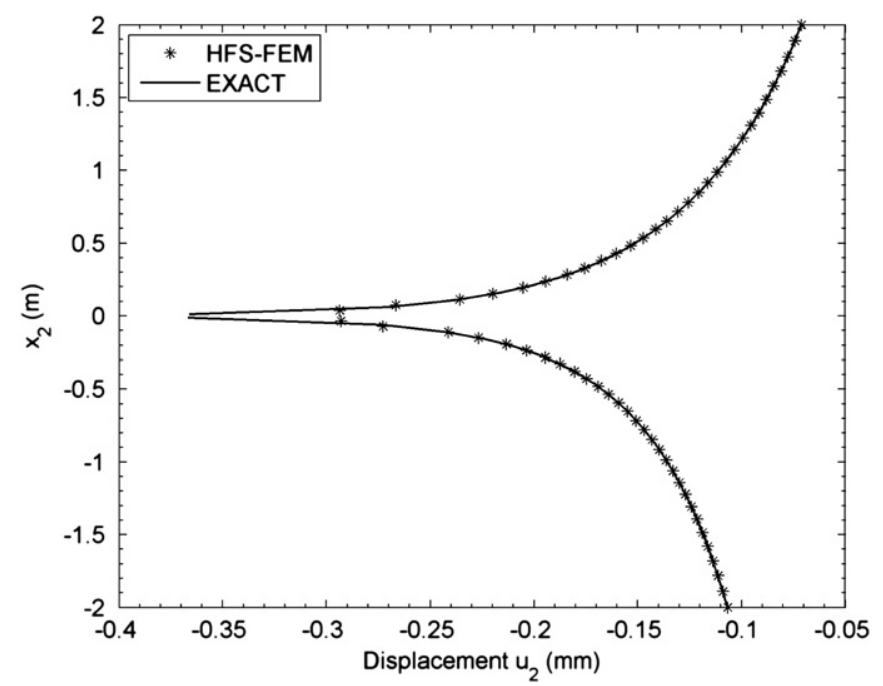

Fig. 8. Variation of displacement $u_{2}$ along the gradation direction $x_{1}=0$.

only, the displacement component $u_{2}$ along the horizontal axis $x_{2}=0$ is symmetric about $x_{2}$-axis, and it is no longer symmetric along the gradation direction. At the same time, it can be seen from Fig. 8 that at any point $x_{2}\left(x_{2}>0\right)$, the amplitude of $u_{2}$ is smaller than that at its mirror point $\left(-x_{2}\right)$. The reason is that the graded variation of material property toward the positive direction of $x_{2}$ causes the material in region $x_{2}>0$ to be stiffer than that in region $x_{2}<0$.

The distributions of stress component $\sigma_{11}$ along two coordinate axes are respectively illustrated in Fig. 9 and 10. Due to the singularity of stress at the origin point of the rectangular coordinate system, there is a significant difference between the theoretical solution and the numerical results from the HFS-FEM in the neighborhood of the origin. However, the results from proposed model agree well with the theoretical results for the points $\left|x_{2}\right|>0.2$. Thus the present hybrid finite model is verified. Besides, numerical results and analytical solutions at selected points are tabulated in Table 1 for more clear comparison.

Finally, the contours of the displacement field in the circle domain are plotted in Fig. 11 and 12. We can see that the

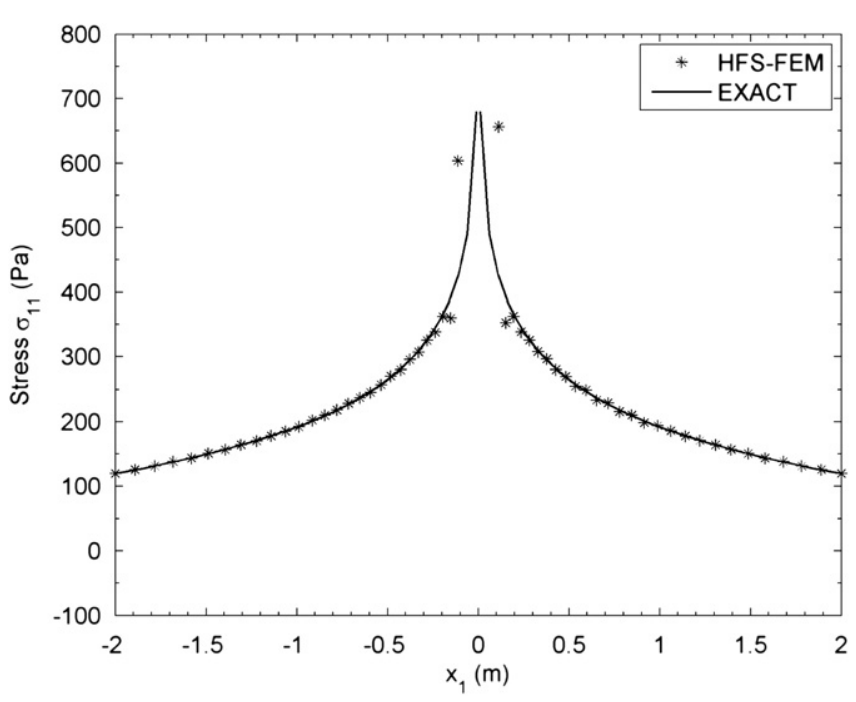

Fig. 9. Variation of stress component $\sigma_{11}$ along the axis $x_{2}=0$.

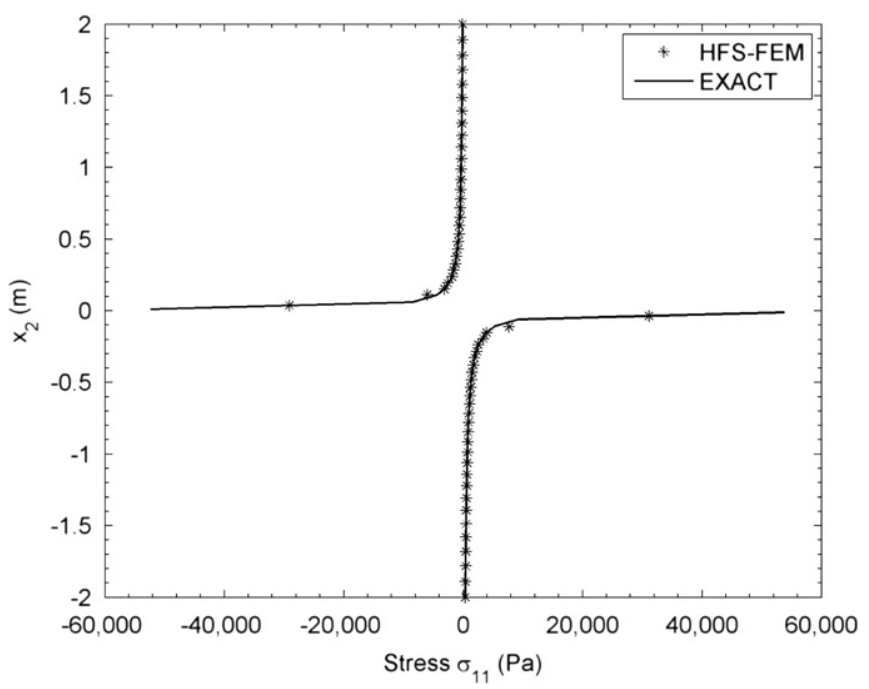

Fig. 10. Variation of stress component $\sigma_{11}$ along the gradation direction $x_{1}=0$.

Table 1

Numerical results and analytical results at selected points in example 1.

\begin{tabular}{llllll}
\hline \multirow{2}{*}{ Location } & \multicolumn{2}{l}{$u_{2}(\mathrm{~mm})$} & & \multicolumn{2}{l}{$\sigma_{11}(\mathrm{~Pa})$} \\
\cline { 2 - 3 } & HFS-FEM & EXACT & & HFS-FEM & \multicolumn{1}{l}{ EXACT } \\
\hline$(0,2.0000)$ & $-7.1146 \mathrm{E}-2$ & $-7.1153 \mathrm{E}-2$ & & $-1.0470 \mathrm{E} 2$ & $-1.0935 \mathrm{E} 2$ \\
$(0,1.0618)$ & $-1.0754 \mathrm{E}-1$ & $-1.0755 \mathrm{E}-1$ & & $-2.9183 \mathrm{E} 2$ & $-2.9605 \mathrm{E} 2$ \\
$(0,0.1939)$ & $-2.0517 \mathrm{E}-1$ & $-2.0519 \mathrm{E}-1$ & & $-2.5877 \mathrm{E} 3$ & $-2.3670 \mathrm{E} 3$ \\
$(0,-2.0000)$ & $-1.0671 \mathrm{E}-1$ & $-1.0673 \mathrm{E}-1$ & & $3.5594 \mathrm{E} 2$ & $3.5751 \mathrm{E} 2$ \\
$(0,-1.0618)$ & $-1.3309 \mathrm{E}-1$ & $-1.3311 \mathrm{E}-1$ & & $6.6809 \mathrm{E} 2$ & $6.6943 \mathrm{E} 2$ \\
$(0,-0.1939)$ & $-2.1327 \mathrm{E}-1$ & $-2.1331 \mathrm{E}-1$ & & $3.3685 \mathrm{E} 3$ & $3.0977 \mathrm{E} 3$ \\
$(2.0000,0)$ & $-5.9903 \mathrm{E}-2$ & $-5.9919 \mathrm{E}-2$ & & $1.1992 \mathrm{E} 2$ & $1.1984 \mathrm{E} 2$ \\
$(1.0618,0)$ & $-9.2732 \mathrm{E}-2$ & $-9.2748 \mathrm{E}-2$ & & $1.8609 \mathrm{E} 2$ & $1.8550 \mathrm{E} 2$ \\
$(0.1939,0)$ & $-1.8263 \mathrm{E}-1$ & $-1.8266 \mathrm{E}-1$ & & $3.6221 \mathrm{E} 2$ & $3.6531 \mathrm{E} 2$ \\
$(-2.0000,0)$ & $-5.9903 \mathrm{E}-2$ & $-5.9919 \mathrm{E}-2$ & & $1.1992 \mathrm{E} 2$ & $1.1984 \mathrm{E} 2$ \\
$(-1.0618,0)$ & $-9.2732 \mathrm{E}-2$ & $-9.2748 \mathrm{E}-2$ & & $1.8503 \mathrm{E} 2$ & $1.8550 \mathrm{E} 2$ \\
$(-0.1939,0)$ & $-1.8263 \mathrm{E}-1$ & $-1.8266 \mathrm{E}-1$ & $3.6166 \mathrm{E} 2$ & $3.6531 \mathrm{E} 2$ \\
\hline
\end{tabular}

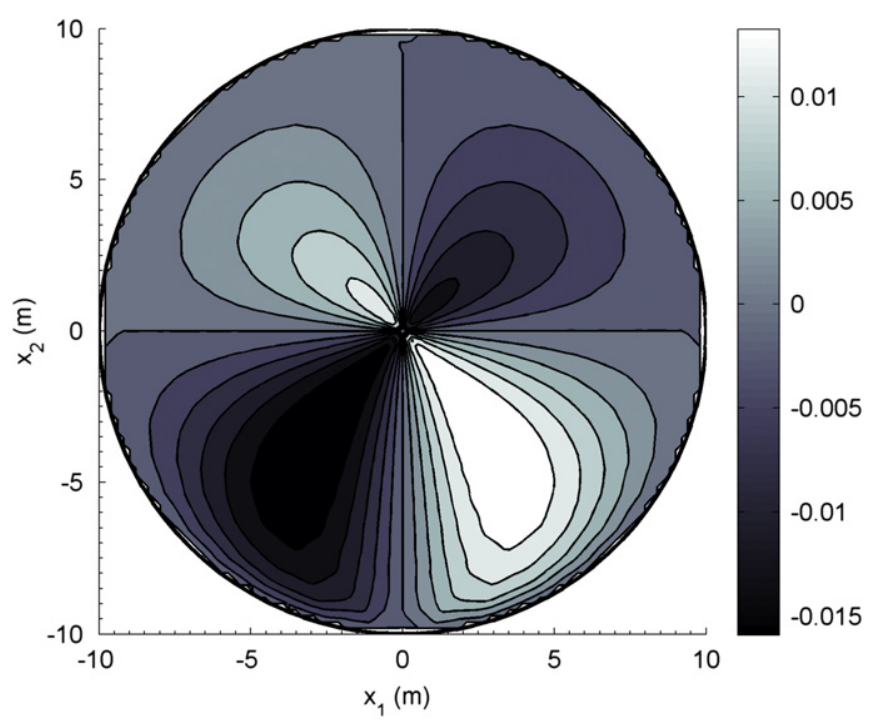

Fig. 11. Distribution of displacement $u_{1}$ over the circle. 


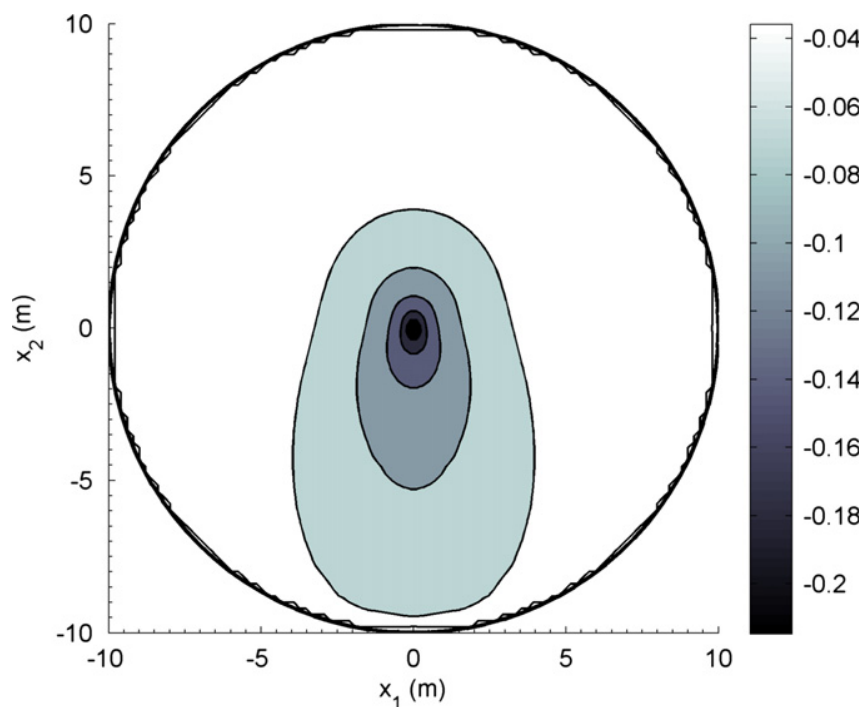

Fig. 12. Distribution of displacement $u_{2}$ over the circle.

distribution of displacement $u_{1}$ shows same symmetric and nonsymmetric properties as that of displacement $u_{2}$, due to the variation in the gradation of material property with respect to $x_{2}$.

\subsection{Functionally graded beam}

In this example we consider an elastic cantilever beam as shown in Fig. 13, made of a two-phase functionally graded composite. The corresponding material elastic modules are respectively $E_{1}=427 \mathrm{GPa}$ and $E_{2}=70 \mathrm{GPa}$. Provided that the elastic parameters are assumed to vary quadratically through the thickness, according to Eq. (47) the graded parameters can be determined by

$\mu_{0}=28 \mathrm{GPa}, \quad \beta_{2}=0.49 \mathrm{~mm}^{-1}$

such that the upper and lower surfaces of the beam are fully enriched with two constituent phases.

In the analysis with the proposed formulation, under the assumption of plane strain deformation, the beam is modeled with 8 and 12 hybrid elements respectively. For the purpose of comparison, ABAQUS is used to generate the corresponding numerical results. In the analysis with ABAQUS the beam is divided into 12 and 20 layers along the graded direction, and in each element a constant Young's modulus taken at the element centroid is employed. Fig. 14 shows the two typical types of mesh configuration used in the present HFS-FEM and ABAQUS.

The displacement distributions in the upper and right surfaces of the functionally graded beam are displayed in Figs. 15 and 16

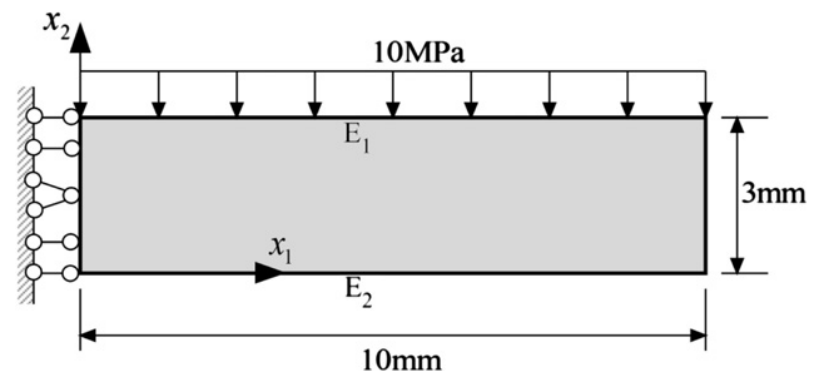

Fig. 13. Functionally graded beam under uniform pressure.

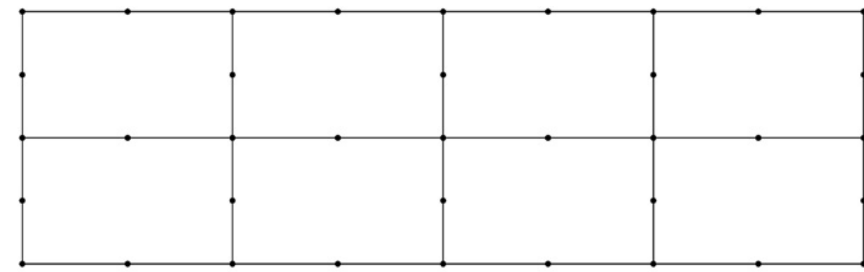

a Element mesh used in HFS-FEM

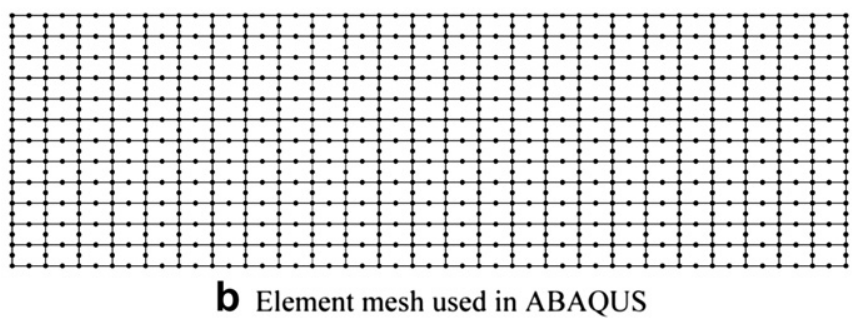

Fig. 14. Element meshes used in the present HFS-FEM and ABAQUS.

respectively, in which $2 \times 4$ elements are used. Good agreement is observed between the results from the present HFS-FEM using 8 graded elements and those from ABAQUS with $12 \times 25$ conventional quadratic quadrilateral elements. The results of two typical homogeneous beams (one with $E_{1}=427 \mathrm{GPa}$ and the other with $E_{2}=70 \mathrm{GPa}$ ) are also presented in the two figures to illustrate the advantage of FGMs over conventional homogeneous materials. In general, the results of a homogeneous beam can be regarded as the upper and lower limits of deformation of a functionally graded beam. Further, we can see from Fig. 15 that there is a common deformation center $\left(u_{1}=0\right)$ for the two homogeneous beams, and the location of the deformation center is the geometric center of the cross-section. However, the graded effect of FGM can move that location up (see Fig. 15). This is because in the FMG beam under consideration the upper half of the beam has greater stiffness than the lower half.

To investigate the stress distribution due to the graded effect of FGM, the normal stress component $\sigma_{11}$ over the middle crosssection $\left(x_{1}=5\right)$ is evaluated and the corresponding results are displayed in Fig. 17. It is evident from Fig. 17 that the results from

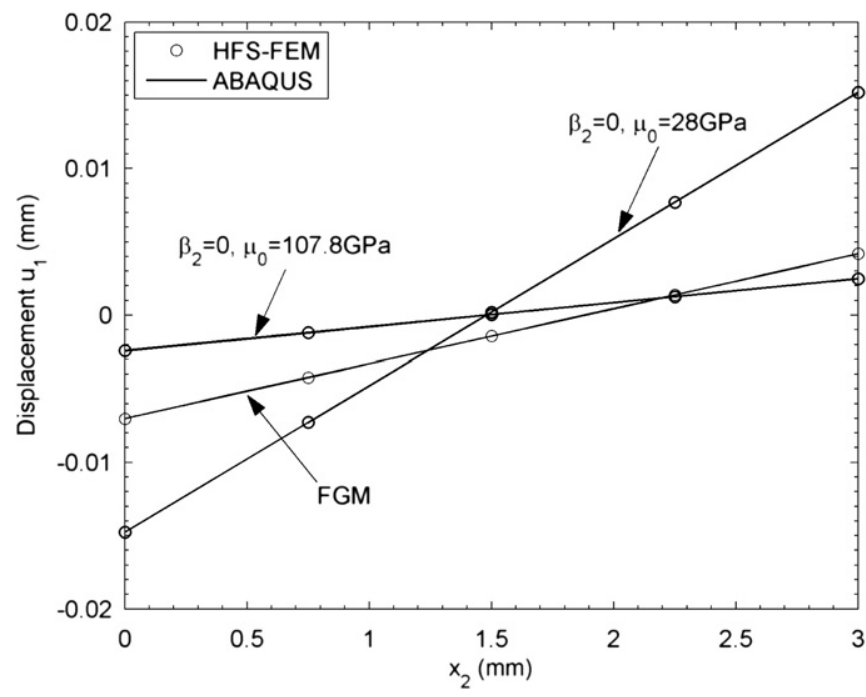

Fig. 15. Horizontal displacement over the right surface of the functionally graded beam. 


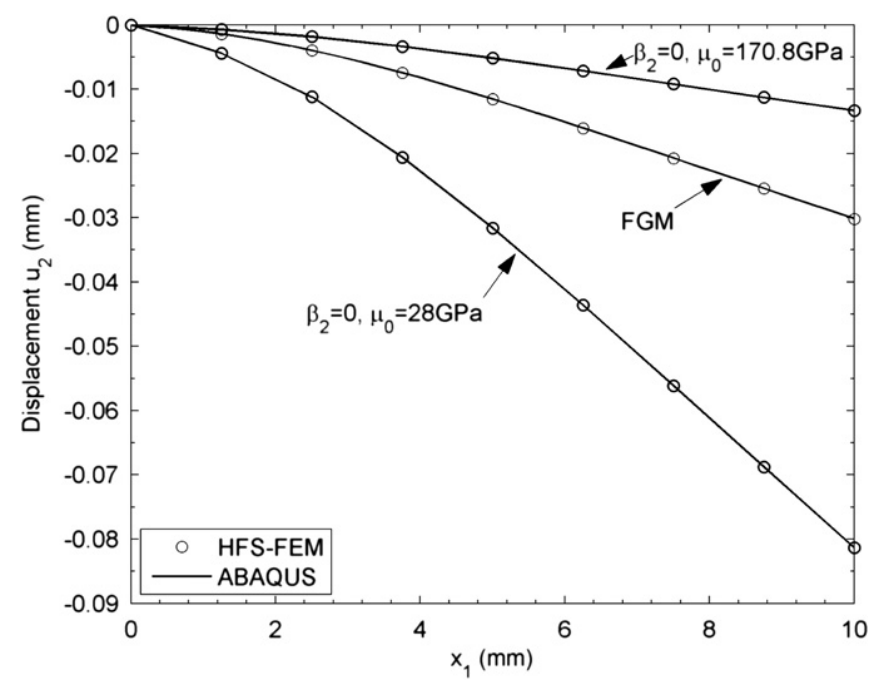

Fig. 16. Vertical displacement over the upper surface of the functionally graded beam.

the refined mesh of both the present HFS-FEM and ABAQUS are not obviously different from those obtained with the coarse mesh, because of the relatively small stress gradation over the middle cross-section at $x_{1}=5$. In contrast, over the cross-section at the fixed end the stresses change dramatically. In this case, mesh refinement does produce better numerical accuracy. For example, at the mid-point of the cross-section at the fixed end, the ABAQUS with 20 material layer definitions gives $\sigma_{11}=-96.57 \mathrm{MPa}$, whereas, the present hybrid model with coarse mesh $(2 \times 4$ elements $)$ and refined mesh $(3 \times 6$ elements $)$ respectively gives $\sigma_{11}=-106.4 \mathrm{MPa}$ and $-97.02 \mathrm{MPa}$. It is obvious that many fewer elements are used in the present HFS-FEM than in ABAQUS. The proposed HFS-FEM is therefore more efficient than the standard FEM and is easy to implement in the analysis of functionally graded structures, as the final stiffness matrix is also symmetric and sparse. Results from ABAQUS with different meshes are also displayed in Fig. 17, from which we observe that the stresses at the upper and lower surfaces become more similar to those from the present HFS-FEM as the number of material layers increases. Thus the correctness of the present hybrid model is again demonstrated,

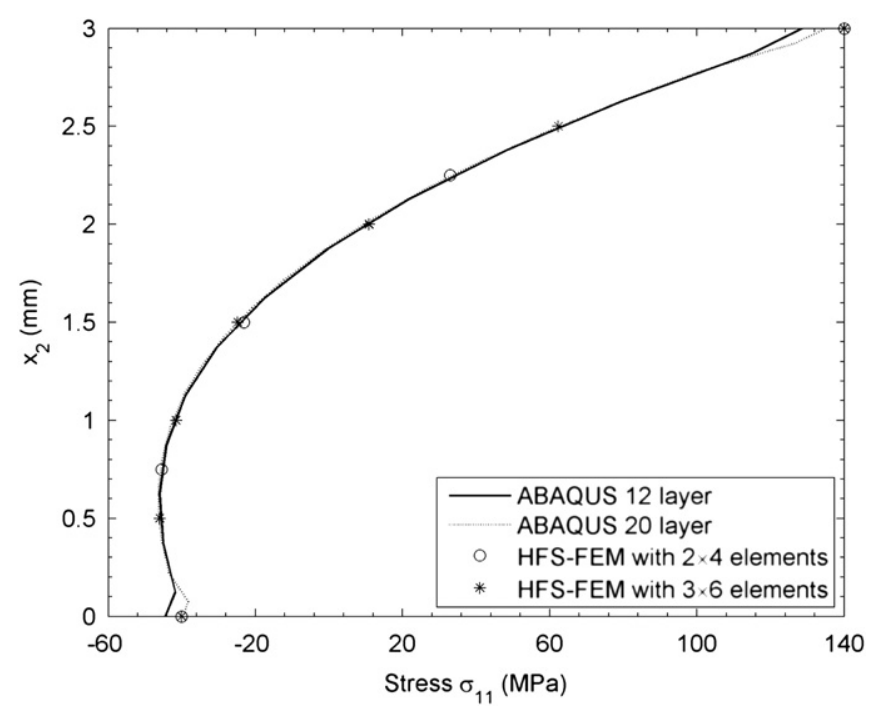

Fig. 17. Stress variation along the line $x_{1}=5$. and it is evident that one can use a relatively coarse mesh to produce numerical results with acceptable accuracy. A similar conclusion on the HFS-FEM was derived in authors' previous works by use of proper fundamental solutions (Wang and Qin, 2009, 2010a,b, 2011).

To illustrate the advantages of FGM over conventional homogeneous materials, the normal stress distribution over the crosssection at the mid-point $\left(x_{1}=5\right)$ of the beam is presented and compared with those of homogeneous materials. It can be observed from Fig. 18 that the graded variation of material properties can significantly change the stress distribution. In contrast to the almost linear distribution of stress $\sigma_{11}$ for homogeneous materials, the FGM under consideration produces an obvious nonlinear stress feature over the cross-section of beam, and simultaneously the value of axis normal stress decreases on the lower surface and increases on the upper surface. This demonstrates that FGMs can be designed to adapt to practical requirements.

Finally, the contour plots of the vertical displacement and axis normal stress in the solution domain are given in Fig. 19 to show their global distribution. From Fig. 19, it can be seen that the vertical displacement shows slight nonlinearity and the axis normal stress shows more complex variation in the region near the clamped end of the beam.

\subsection{Functionally graded link bar}

To further assess the performance of the proposed hybrid formulation, we now consider the isotropic functionally graded link bar illustrated in Fig. 20. The bar has unit thickness and is subjected to $10 \mathrm{MPa}$ axial tension load at the right end. The basic FGM constituents are titanium monoboride (TiB) and commercially pure titanium (CP Ti) whose elastic properties are

$E_{\mathrm{TiB}}=375 \mathrm{GPa}, \quad E_{\mathrm{Ti}}=107 \mathrm{GPa}$

from which we have

$\mu_{0}=42.8 \mathrm{GPa}, \quad \beta_{2}=0.12458 \mathrm{~mm}^{-1}$

For the sake of simplicity and comparison, we, consider first a homogeneous material, which is obtained simply by setting $\beta_{2}=0$. Due to the symmetry with respect to the $x_{1}$-axis (see

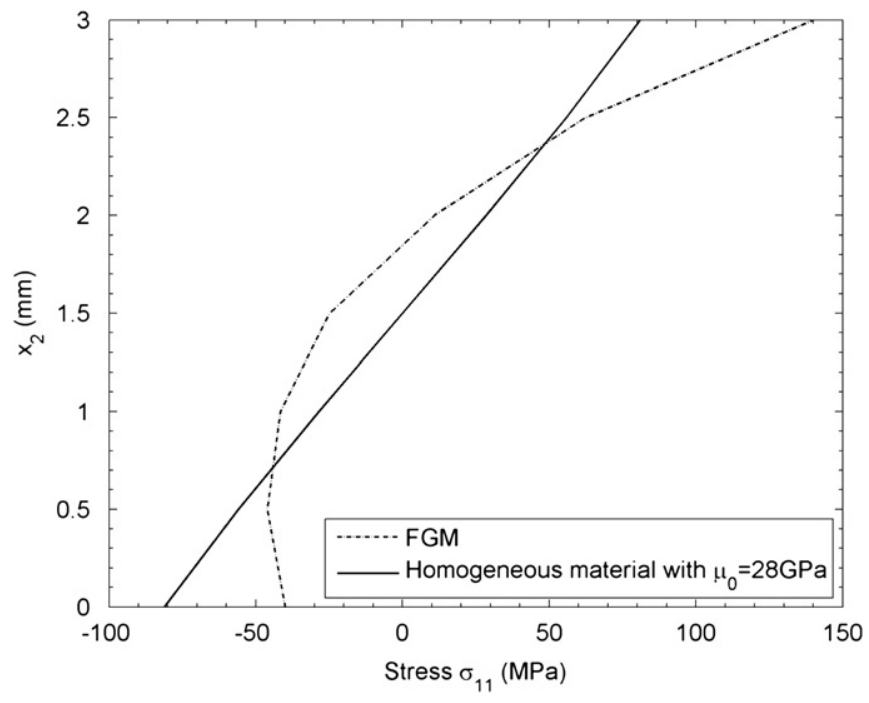

Fig. 18. Comparison of stress variation between homogeneous and graded materials. 


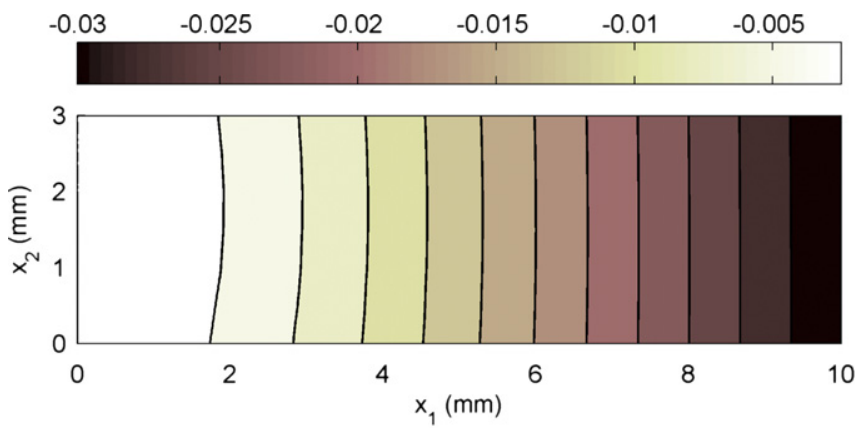

a displacement $u_{2}$

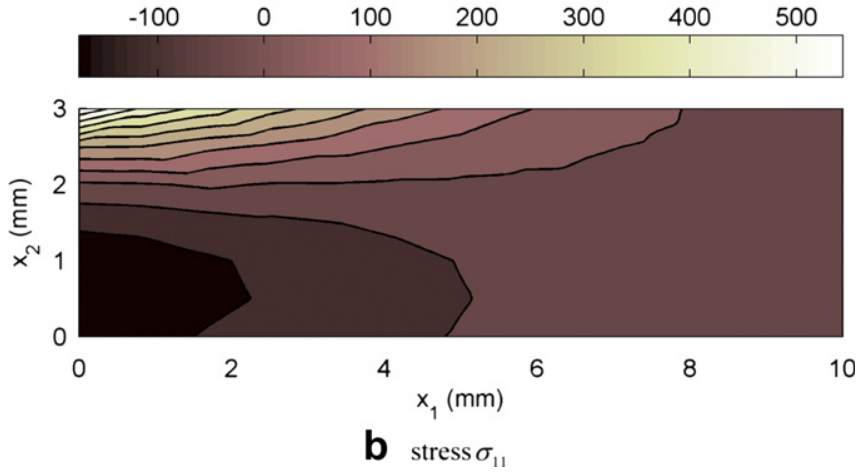

Fig. 19. Contour maps of displacement and stress of the beam.

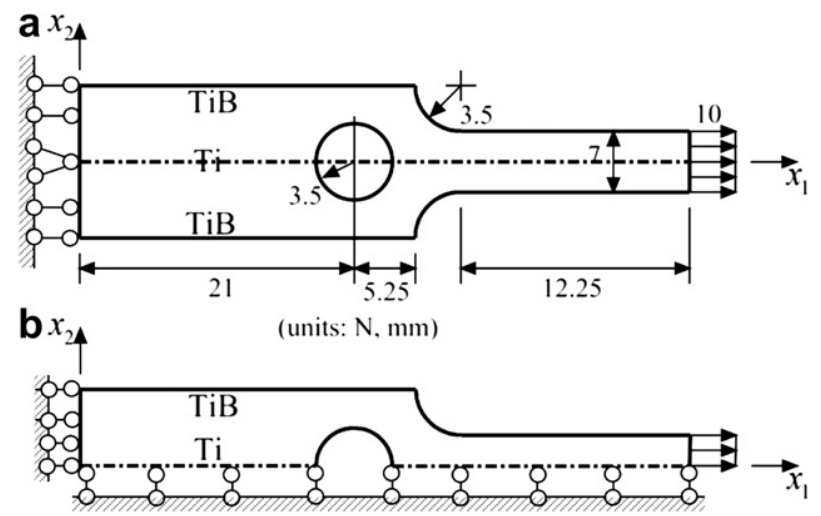

Fig. 20. FGM link bar (a) geometry and boundary condition (b) symmetric computing model.

Fig. 20a), only the upper half of the link bar is modeled (see Fig. 20b). The element mesh used (146 elements and 515 nodes) is shown in Fig. 21. The stresses $\sigma_{11}$ along two circular rims centered at $(21,0)$ and $(29.75,7)$ are calculated and the corresponding results are plotted in Fig. 22 and 23. In these two figures, the numerical results obtained using ABAQUS are also presented for the purpose of comparison. In the calculation with ABAQUS, both the coarse mesh shown in Fig. 21, which is also used in the present HFS-FEM,

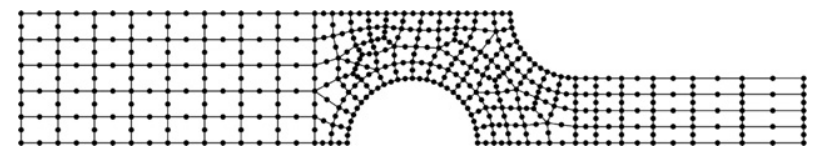

Fig. 21. Element mesh used in the present HFS-FEM.

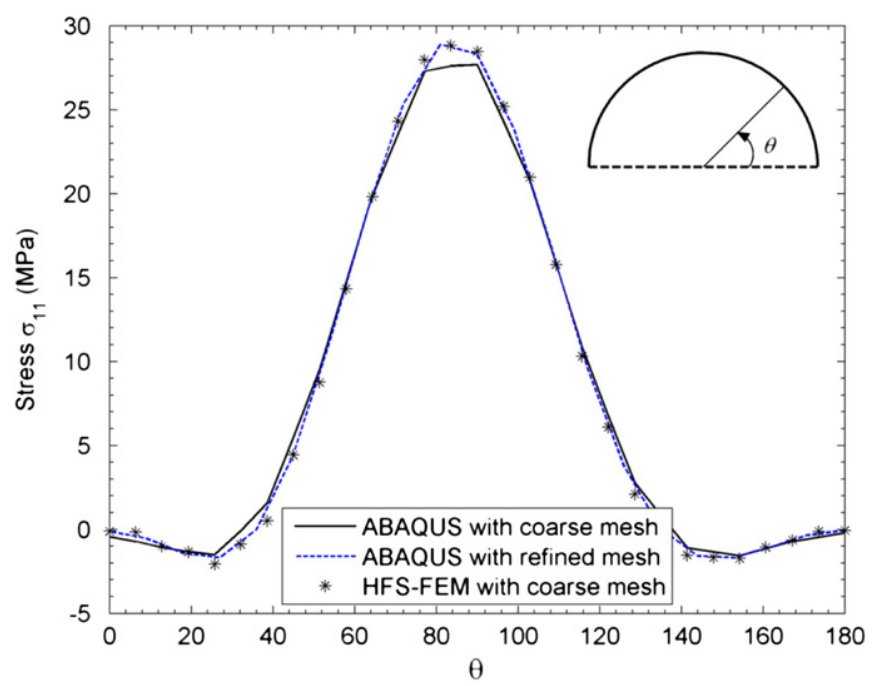

Fig. 22. The stress $\sigma_{11}$ along the rim of the circle centered at $(21,0)$ for the case of homogeneous material.

and a refined mesh including 276 elements and 933 nodes are employed to assess the numerical accuracy of the proposed method. It can be seen that the present method achieves better numerical accuracy than ABAQUS when the same element mesh as shown in Fig. 21 is used. Simultaneously, we observe that there is stress concentration on the two circular-arc boundaries. The stress concentration factors (SCFs) are respectively 2.884 and 2.132 , which will be used to investigate the graded effect of FGM.

Subsequently, the effect of graded material properties on stress distribution and the corresponding SCFs are investigated by comparing the results of a FGM link bar with those of a homogeneous link bar. With the mesh division displayed in Fig. 21, the axial stress variations on the boundaries of the circles centered at $(21,0)$ and $(29.75,7)$ are displayed in Fig. 24 and 25, respectively. The results are obtained using the element mesh displayed in Fig. 21. For the circle centered at $(21,0)$, we note from Fig. 24 that the stress in FGM is smaller than that in the homogeneous material near the area around the point $(21.392,3.478)$ at which the maximum stress

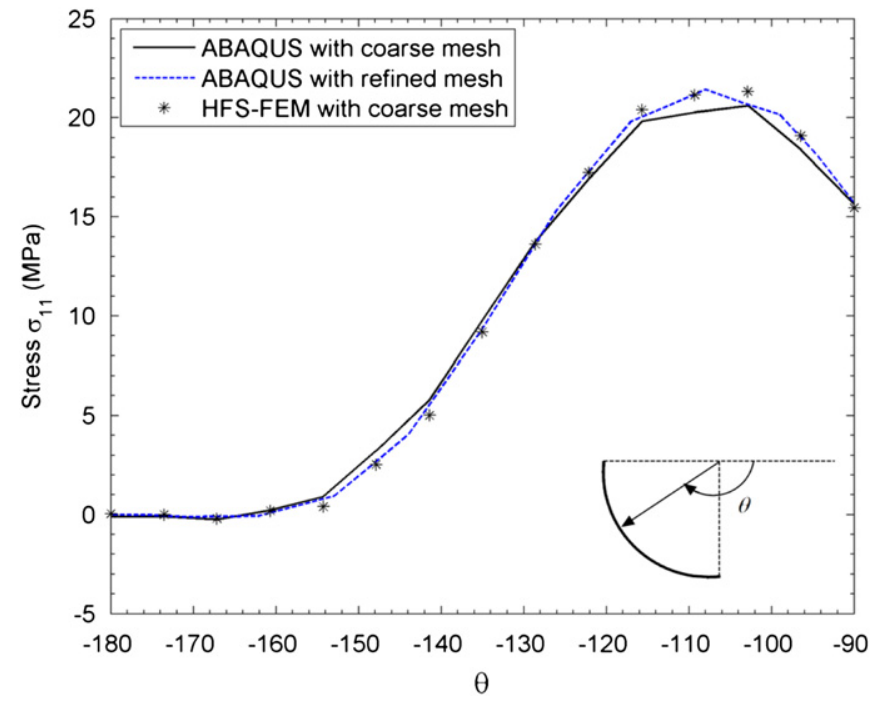

Fig. 23. The stress $\sigma_{11}$ along the rim of the circle centered at $(29.75,7)$ for the case of homogeneous material. 


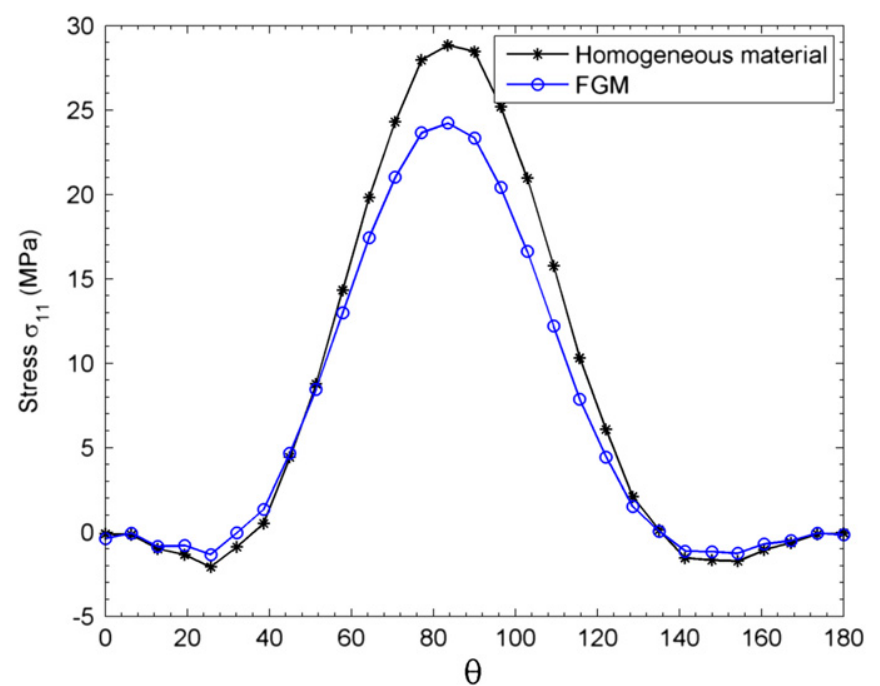

Fig. 24. The stress $\sigma_{11}$ along the rim of the circle centered at $(21,0)$.

is $24.22 \mathrm{MPa}$. The point corresponds to the rotational angle $83.57^{\circ}$ measured anticlockwise (see Fig. 22). It is found from Fig. 24 that the SCF in the FGM link bar is 2.422, which is smaller than that in the homogeneous link bar ( $\mathrm{SCF}=2.884)$. For the circle centered at $(29.75,7)$, the maximum stress $(26.43 \mathrm{MPa})$ occurs at the point $(28.971,3.588)$ which corresponds to the rotational angle $-102.86^{\circ}$ measured anticlockwise (see Fig. 23). The corresponding SCF is 2.643 , which is larger than that of the homogeneous material $(\mathrm{SCF}=2.132)$. In contrast to the maximum stress in the homogeneous link bar which occurs at $(21,0)$, the maximum stress in the FGM link bar occurs at the circle rim centered at $(29.75,7)$ and it is smaller than that in the homogeneous link bar (28.84 MPa). Similar results have been found in exponentially graded link bars (Ching and Yen, 2005; Kim and Paulino, 2002). This is reasonable because the elastic module with quadratic variation is similar to that with exponential variation (see Fig. 26). Finally, the stress concentration factors for the cases of FGM and homogeneous materials are listed in Table 2 to show clearly the effect of graded parameter.

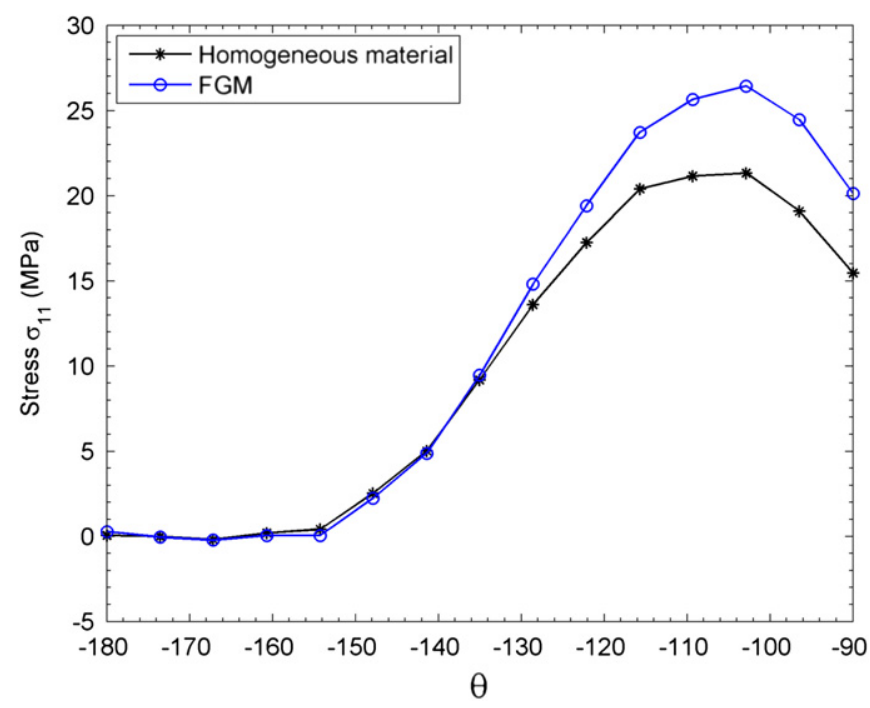

Fig. 25. The stress $\sigma_{11}$ along the rim of the circle centered at $(29.75,7)$.

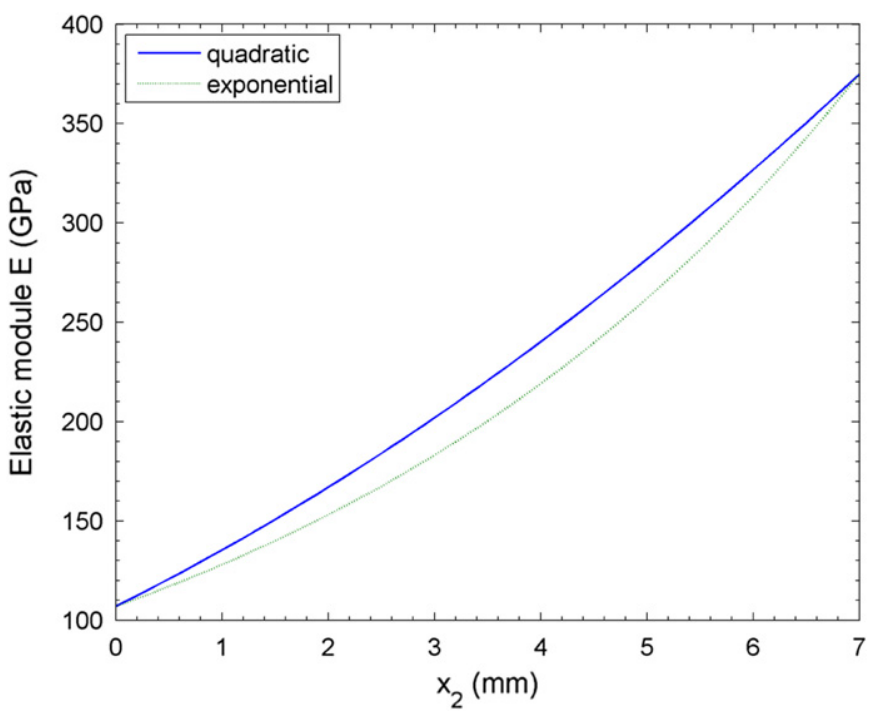

Fig. 26. Comparison of different material variations along the graded direction.

Table 2

Stress concentration factors for FGM and homogeneous materials in example 3.

\begin{tabular}{lll}
\hline \multirow{2}{*}{ Location } & \multicolumn{2}{l}{ Stress concentration factor } \\
\cline { 2 - 3 } & FGM & Homogeneous materials \\
\hline Circle centered at $(21,0)$ & 2.422 & 2.884 \\
Circle centered at $(29.75,7)$ & 2.643 & 2.132 \\
\hline
\end{tabular}

\section{Conclusions}

In this work, we present HFS-FEM models for analyzing 2D functionally graded solids under mechanical loads. In the HFS-FEM model, the fundamental solution of graded materials is used to interpolate the intra-element displacement and stress fields, and the shape functions used in conventional FEM are employed to approximate the frame fields defined on the element boundary only. The linkage of these two fields is forced by the weak hybrid functional constructed in this work. The proposed graded hybrid element is then used for displacement and stress analysis of FGMs. The material definition at element level is permitted to keep its natural variation over the element (quadratic variation in this work). Results obtained from the proposed finite element formulation are compared with those obtained from analytical methods or ABAQUS and good agreement between them is observed. It is clear that the deformation and stress fields of functionally graded solids differ substantially from those of their homogeneous counterparts. The computed results indicate that graded elastic properties can be tailored to lower deflection, redistribute stress fields and minimize stress concentration. The present hybrid model is shown to be capable of capturing these features of FGMs and to be more efficient and accurate than classical commercial FE codes in the analysis of functionally graded structures.

\section{References}

Ayhan, A.O., 2009. Three-dimensional mixed-mode stress intensity factors for cracks in functionally graded materials using enriched finite elements. International Journal of Solids and Structures 46, 796-810.

Carrera, E., Brischetto, S., Robaldo, A., 2008. Variable kinematic model for the analysis of functionally graded material plates. AIAA Journal 46, 194-203.

Ching, H.K., Yen, S.C., 2005. Meshless local Petrov-Galerkin analysis for 2D functionally graded elastic solids under mechanical and thermal loads. Composites Part B: Engineering 36, 223-240. 
Dai, K.Y., Liu, G.R., Han, X., Lim, K.M., 2005. Thermomechanical analysis of functionally graded material (FGM) plates using element-free Galerkin method. Computers \& Structures 83, 1487-1502.

Fairweather, G., Karageorghis, A., 1998. The method of fundamental solutions for elliptic boundary value problems. Advances in Computational Mathematics 9, 69-95.

Kim, J.H., Paulino, G.H., 2002. Isoparametric graded finite elements for nonhomogeneous isotropic and orthotropic materials. Journal of Applied Mechanics 69 502-514.

Manolis, G.D., Shaw, R.P., 1996. Green's function for the vector wave equation in a mildly heterogeneous continuum. Wave Motion 24, 59-83.

Marin, L., Lesnic, D., 2007. The method of fundamental solutions for nonlinear functionally graded materials. International Journal of Solids and Structures 44 6878-6890.

Qin, Q.H., 1993. Nonlinear-analysis of reissner plates on an elastic-foundation by the BEM. International Journal of Solids and Structures 30, 3101-3111.

Qin, Q.H., 2000. The Trefftz Finite and Boundary Element Method. WIT Press, Southampton.

Reddy, J.N., 2000. Analysis of functionally graded plates. International Journal for Numerical Methods in Engineering 47, 663-684.

Sladek, V., Sladek, J., Tanaka, M., Zhang, C., 2005. Local integral equation method for potential problems in functionally graded anisotropic materials. Engineering Analysis with Boundary Elements 29, 829-843.

Sutradhar, A., Paulino, G.H., 2004. The simple boundary element method for transient heat conduction in functionally graded materials. Computer Methods in Applied Mechanics and Engineering 193, 4511-4539.

Wang, H., Qin, Q.H., 2008. Meshless approach for thermo-mechanical analysis of functionally graded materials. Engineering Analysis with Boundary Elements $32,704-712$
Wang, H., Qin, Q.H., 2009. Hybrid FEM with fundamental solutions as trial functions for heat conduction simulation. Acta Mechanica Solida Sinica 22, 487-498.

Wang, H., Qin, Q.H., 2010a. FE approach with green's function as internal trial function for simulating bioheat transfer in the human eye. Archives of Mechanics 62, 493-510.

Wang, H., Qin, Q.H., 2010b. Fundamental-solution-based finite element model for plane orthotropic elastic bodies. European Journal of Mechanics-A/Solids 29, 801-809.

Wang, H., Qin, Q.H., 2011. Fundamental-solution-based hybrid FEM for plane elasticity with special elements. Computational Mechanics 48, 515-528.

Wang, H., Qin, Q.H., Kang, Y.L., 2005. A new meshless method for steady-state heat conduction problems in anisotropic and inhomogeneous media. Archive of Applied Mechanics 74, 563-579.

Wang, H., Qin, Q.H., Kang, Y.L., 2006. A meshless model for transient heat conduction in functionally graded materials. Computational Mechanics 38, 51-60.

Young, D.L., Chiu, C.L., Fan, C.M., Tsai, C.C., Lin, Y.C., 2006. Method of fundamental solutions for multidimensional stokes equations by the dual-potential formulation. European Journal of Mechanics - B/Fluids 25, 877-893.

Yuan, Z.F., Yin, H.M., 2011. elastic green's functions for a specific graded material with a quadratic variation of elasticity. Journal of Applied Mechanics 78, 021021-021026.

Zhang, C., Cui, M., Wang, J., Gao, X.W., Sladek, J., Sladek, V., 2011. 3D crack analysis in functionally graded materials. Engineering Fracture Mechanics 78, 585-604.

Zhang, G.M., Batra, R.C., 2007. Wave propagation in functionally graded materials by modified smoothed particle hydrodynamics (MSPH) method. Journal of Computational Physics 222, 374-390. 